\title{
Cohomologies of Sasakian groups and Sasakian solvmanifolds
}

\author{
Hisashi Kasuya ${ }^{1}$
}

Received: 4 December 2014 / Accepted: 28 October 2015 / Published online: 7 November 2015

(C) Fondazione Annali di Matematica Pura ed Applicata and Springer-Verlag Berlin Heidelberg 2015

\begin{abstract}
We show certain symmetry of the dimensions of cohomologies of the fundamental groups of compact Sasakian manifolds by using the Hodge theory of twisted basic cohomology. As applications, we show that the polycyclic fundamental groups of compact Sasakian manifolds are virtually nilpotent, and Sasakian solvmanifolds are finite quotients of Heisenberg nilmanifolds.
\end{abstract}

Keywords Sasakian group $\cdot$ Hodge theory $\cdot$ Solvmanifold $\cdot$ Basic cohomology

Mathematics Subject Classification $53 \mathrm{C} 25 \cdot 53 \mathrm{C} 30 \cdot 58 \mathrm{~A} 14$

\section{Introduction}

The purpose of this paper is to study the fundamental groups of compact Sasakian manifolds. Sasakian manifolds constitute an odd-dimensional counterpart of the class of Kähler manifolds. A Riemannian manifold $(M, g)$ is a Sasakian manifold if the cone metric on the cone manifold $M \times \mathbb{R}_{+}$is a Kähler metric. The fundamental groups of compact Kähler manifolds satisfy various properties. Since the first cohomology of manifolds and the first group cohomology of their fundamental groups are isomorphic, the first group cohomology of the fundamental group of a compact Kähler manifold was studied precisely by using the Hodge theory. In this paper, we study the first cohomologies of the fundamental groups of compact Sasakian manifolds for one-dimensional representations by using the Hodge theory of the basic cohomology.

Let $\Gamma$ be a group. We denote by $\mathcal{C}(\Gamma)$ the space of characters $\Gamma \rightarrow \mathrm{GL}_{1}(\mathbb{C})$ which can be factored as

Hisashi Kasuya

kasuya@math.titech.ac.jp

1 Department of Mathematics, Tokyo Institute of Technology, 2-12-1 Ookayama, Meguro-ku, Tokyo 152-8551, Japan 


$$
\Gamma \rightarrow H_{1}(\Gamma, \mathbb{Z}) /(\text { torsion }) \rightarrow \mathrm{GL}_{1}(\mathbb{C})
$$

For $\rho \in \mathcal{C}(\Gamma)$, we denote by $H^{*}\left(\Gamma, \mathbb{C}_{\rho}\right)$ the group cohomology of $\Gamma$ with values in the module associated with the one-dimensional representation $\rho$. Considering the exponential map $\mathbb{C} \rightarrow \mathbb{C}^{*}=\mathrm{GL}_{1}(\mathbb{C})$, we have the surjective map $\mathcal{E}: H^{1}(\Gamma, \mathbb{C}) \rightarrow \mathcal{C}(\Gamma)$. We define the "real" action of $\mathbb{R}^{*}$ on $\mathcal{C}(\Gamma)$ such that for $f_{1}+\sqrt{-1} f_{2} \in H^{1}(\Gamma, \mathbb{C})$ with $f_{1}, f_{2} \in H^{1}(M, \mathbb{R})$, the action is given by

$$
t \cdot \mathcal{E}\left(f_{1}+\sqrt{-1} f_{2}\right)=\mathcal{E}\left(t f_{1}+\sqrt{-1} f_{2}\right)
$$

for $t \in \mathbb{R}^{*}$.

In this paper, we prove the " $\mathbb{R}^{*}$-symmetry" of cohomologies of the fundamental group of a compact Sasakian manifold.

Theorem 1.1 Let $M$ be a compact Sasakian manifold. Then for each $\rho \in \mathcal{C}\left(\pi_{1}(M)\right)$ and $t \in \mathbb{R}^{*}$, we have

$$
\operatorname{dim} H^{*}\left(\pi_{1}(M), \mathbb{C}_{\rho}\right)=\operatorname{dim} H^{*}\left(\pi_{1}(M), \mathbb{C}_{t \cdot \rho}\right) .
$$

For a group $\Gamma$, we consider the set

$$
\mathcal{J}_{k}(\Gamma)=\left\{\rho \in \mathcal{C}(\Gamma) \mid \operatorname{dim} H^{1}\left(\Gamma, \mathbb{C}_{\rho}\right) \geq k\right\} .
$$

For $\mathcal{C}\left(\pi_{1}(M)\right) \ni \rho=\mathcal{E}\left(f_{1}+\sqrt{-1} f_{2}\right)$ with $f_{1}, f_{2} \in H^{1}(M, \mathbb{R}), \mathcal{E}\left(f_{1}+\sqrt{-1} f_{2}\right)$ is fixed by the $\mathbb{R}^{*}$-action if and only if $f_{1}=0$ (equivalently $\rho$ is unitary). Hence, we have the following corollary.

Corollary 1.2 Let $M$ be a compact Sasakian manifold. If there exists a non-unitary character $\rho: \pi_{1}(M) \rightarrow \mathrm{GL}_{1}(\mathbb{C})$ satisfying $H^{1}\left(\pi_{1}(M), \mathbb{C}_{\rho}\right) \geq k$, then the set $\mathcal{J}_{k}\left(\pi_{1}(M)\right)$ is an infinite set.

A group $\Gamma$ is polycyclic if it admits a sequence

$$
\Gamma=\Gamma_{0} \supset \Gamma_{1} \supset \cdots \supset \Gamma_{k}=\{e\}
$$

of subgroups such that each $\Gamma_{i}$ is normal in $\Gamma_{i-1}$ and $\Gamma_{i-1} / \Gamma_{i}$ is cyclic. By Corollary 1.2, we prove the following result which is analogous to the Arapura-Nori's result in [2].

Corollary 1.3 Let $M$ be a compact Sasakian manifold. Suppose that the fundamental group $\pi_{1}(M)$ is polycyclic. Then $\pi_{1}(M)$ is virtually nilpotent, i.e., it admits a nilpotent subgroup of finite index.

Remark 1 In [7], Chen proves that the solvable fundamental group of a compact Sasakian manifold is virtually nilpotent. Chen's result is based on Campana's results on Kähler orbifolds [5]. Campana uses algebraic and complex analytic geometrical techniques. In this paper, we only use standard differential geometrical techniques on transversely Kähler foliations. This gives a technical advantage. In Chen's proof, we need the existence of quasi-regular Sasakian structure on a Sasakian manifold. On the other hand, in this paper, we do not use quasi-regularity.

We consider nilmanifolds and solvmanifolds. Solvmanifolds (resp. nilmanifolds) are compact homogeneous spaces of solvable (resp. nilpotent) Lie groups. It is known that every nilmanifold can be represented by $G / \Gamma$ such that $G$ is a simply connected nilpotent Lie group and $\Gamma$ is a lattice in $G$ (see [15]). 
In [6], it is proved that a compact $2 n+1$-dimensional nilmanifold admits a Sasakian structure if and only if it is a Heisenberg nilmanifold $H_{2 n+1} / \Gamma$ where $H_{2 n+1}$ is the $(2 n+1)$ dimensional Heisenberg Lie group and $\Gamma$ is its lattice.

By Corollary 1.3, we can easily extend the result in [6] for solvmanifolds as in [10].

Corollary 1.4 A compact $2 n+1$-dimensional solvmanifold admitting a Sasakian structure is a finite quotient of Heisenberg nilmanifold.

Proof It is known that the fundamental group of a compact solvmanifold is a torsion-free polycyclic group and solvmanifolds with isomorphic fundamental groups are diffeomorphic (see [15]). Hence, by Corollary 1.3 , we can easily show that a compact $2 n+1$-dimensional solvmanifold admitting a Sasakian structure is a finite quotient of a Sasakian nilmanifold. Thus, the Corollary follows from the result in [6].

In particular, we have the following result.

Corollary 1.5 Let $G$ be a $2 n+1$-dimensional simply connected solvable Lie group with a lattice $\Gamma$. We assume that $G$ is completely solvable (i.e., for any $g \in G$, all eigenvalues of the adjoint operator $\operatorname{Ad}_{g}$ are real). Then the compact solvmanifold $G / \Gamma$ admits a Sasakian structure if and only if it is a Heisenberg nilmanifold.

Proof By the Saito's rigidity theorem in [16], if a simply connected completely solvable Lie group contains a nilpotent lattice, then it is nilpotent. Hence, if $G / \Gamma$ admits a Sasakian structure, then by Corollary 1.3 , we can easily show that $G$ is nilpotent. Thus the Corollary follows from the result in [6].

\section{Preliminary}

Let $M$ be a compact smooth manifold and $A^{*}(M)$ the de Rham complex of $M$. For a $\mathbb{C}$-valued closed 1-form $\phi \in A^{r}(M) \otimes \mathbb{C}$, we consider the operator $\phi \wedge: A^{r}(M) \otimes \mathbb{C} \rightarrow A^{r+1}(M) \otimes \mathbb{C}$ of left-multiplication. Define $d_{\phi}=d+\phi \wedge$. Then we have $d_{\phi} d_{\phi}=0$ and hence $\left(A^{*}(M) \otimes \mathbb{C}, d_{\phi}\right)$ is a cochain complex. We denote by $H^{*}(M, \phi)$ the cohomology of $\left(A^{*}(M) \otimes \mathbb{C}, d_{\phi}\right)$. The cochain complex $\left(A^{*}(M) \otimes \mathbb{C}, d_{\phi}\right)$ is considered as the de Rham complex with values in the topologically trivial flat bundle $M \times \mathbb{C}$ with the connection form $\phi$. Hence, the structure of the cochain complex $\left(A^{*}(M) \otimes \mathbb{C}, d_{\phi}\right)$ is determined by the character $\rho_{\phi}: \pi_{1}(M) \rightarrow \mathrm{GL}_{1}(\mathbb{C})$ given by $\rho_{\phi}(\gamma)=\exp \left(\int_{\gamma} \phi\right)$. We have an isomorphism $H^{1}(M, \phi) \cong H^{*}\left(\pi_{1}(M), \mathbb{C}_{\rho_{\phi}}\right)$. The map $H^{1}(M, \mathbb{C}) \ni[\phi] \mapsto \rho_{\phi} \in \mathcal{C}\left(\pi_{1}(M)\right)$ is identified with the map $\mathcal{E}$ as in Introduction. Hence, the action of $\mathbb{R}^{*}$ on $\mathcal{C}\left(\pi_{1}(M)\right)$ as in Introduction is given by

$$
t \cdot \rho_{\phi}=\rho_{t \operatorname{Re} \phi+\sqrt{-1} \operatorname{Im} \phi}
$$

for $t \in \mathbb{R}^{*}$ and $[\phi] \in H^{1}(M, \mathbb{C})$.

\section{Proof of Theorem 1.1}

\subsection{Basic cohomology}

Let $M$ be a compact $(2 n+1)$-dimensional Sasakian manifold with a Sasakian metric $g$ and $\eta$ the contact structure associated with the Sasakian structure. Take $\xi$ the Reeb vector field. Let 
$A^{*}(M)$ be the de Rham complex of $M$. A differential form $\alpha \in A^{*}(M)$ is basic if $\iota \xi \alpha=0$ and $\iota \xi d \alpha=0$. Denote by $A_{B}^{*}(M)$ the differential graded algebra of the basic differential forms on $M$ and denote by $H_{B}^{*}(M, \mathbb{R})\left(\right.$ resp. $\left.H_{B}^{*}(M, \mathbb{C})\right)$ the cohomology of $A_{B}^{*}(M)$ (resp. $A_{B}^{*}(M) \otimes \mathbb{C}$ ). Then it is known that the inclusion $A_{B}^{*}(M) \subset A^{*}(M)$ induces a cohomology isomorphism $H_{B}^{0}(M, \mathbb{R}) \cong H^{0}(M, \mathbb{R})$ and $H_{B}^{1}(M, \mathbb{R}) \cong H^{1}(M, \mathbb{R})$ (see [4]).

Consider the Hodge star operator $*: A^{r}(M) \rightarrow A^{2 n+1-r}(M)$ for the Sasakian metric $g$. We define the transverse Hodge star operator $*_{T}: A_{B}^{r}(M) \rightarrow A_{B}^{2 n-r}(M)$ as $*_{T}(\alpha)=*(\eta \wedge$ $\alpha$ ). Then we have $*_{T}^{2} \alpha=(-1)^{r} \alpha$. Restricting the scalar product $\langle\rangle:, A^{*}(M) \times A^{*}(M) \rightarrow \mathbb{R}$ on the basic forms $A_{B}^{*}(M)$ we consider formal adjoint $\delta_{B}: A_{B}^{r}(M) \rightarrow A_{B}^{r-1}(M)$ of the differential $d$ on $A_{B}^{*}(M)$. Then we have $\delta_{B}=-*_{T} d *_{T}$. Consider the basic Laplacian $\Delta_{B}=d \delta_{B}+\delta_{B} d$. A basic form $\alpha \in A_{B}^{r}(M)$ is harmonic if $\Delta_{B} \alpha=0$. Denote $\mathcal{H}_{B}^{r}(M)=$

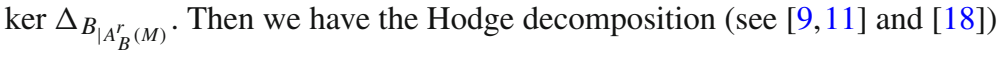

$$
A_{B}^{r}(M)=\mathcal{H}_{B}^{r}(M) \oplus \operatorname{im} d_{\mid A_{B}^{r-1}(M)} \oplus \operatorname{im} \delta_{B_{\mid A_{B}^{r+1}(M)}} .
$$

We have the transverse complex structure on $\operatorname{ker} \iota \xi \subset \bigwedge T M^{*}$, and we obtain the bigrading $A_{B}^{r}(M) \otimes \mathbb{C}=\bigoplus_{p+q=r} A_{B}^{p, q}(M)$ with the bi-differential $d=\partial_{B}+\bar{\partial}_{B}$. We consider the formal adjoint $\partial_{B}^{*}: A_{B}^{p, q}(M) \rightarrow A_{B}^{p-1, q}(M)$ and $\bar{\partial}_{B}^{*}: A_{B}^{p, q}(M) \rightarrow A_{B}^{p, q-1}(M)$ of $\partial_{B}$ and $\bar{\partial}_{B}$ respectively for the restricted Hermitian inner product on $A_{B}^{*}(M) \otimes \mathbb{C}$. Then we have $\partial_{B}^{*}=-*_{T} \bar{\partial}_{B} *_{T}$ and $\bar{\partial}_{B}^{*}=-*_{T} \partial_{B} *_{T}$. Denote $\Delta^{\prime}=\partial_{B} \partial_{B}^{*}+\partial_{B}^{*} \partial_{B}$ and $\Delta^{\prime \prime}=\bar{\partial}_{B} \bar{\partial}_{B}^{*}+\bar{\partial}_{B}^{*} \bar{\partial}_{B}$.

Let $\omega=d \eta$. Then $\omega$ gives a transverse Kähler structure. We can take a complex coordinate system $\left(z_{1}, \ldots, z_{n}\right)$ which is transverse to $\xi$ such that $\omega$ is a Kahler form on $\left(z_{1}, \ldots, z_{n}\right)$. Define the operator $L: A_{B}^{p, q}(M) \rightarrow A_{B}^{p+1, q+1}(M)$ by $L \alpha=\omega \wedge \alpha$ and consider the formal adjoint $\Lambda: A_{B}^{p, q}(M) \rightarrow A_{B}^{p-1, q-1}(M)$ of $L$. We have $\Lambda=-*_{T} L *_{T}$. By using the transverse Kähler geometry, we obtain the following Kähler identity.

\section{Lemma 3.1}

$$
\Lambda \partial_{B}-\partial_{B} \Lambda=\sqrt{-1} \bar{\partial}_{B}^{*} \text { and } \Lambda \bar{\partial}_{B}-\bar{\partial}_{B} \Lambda=-\sqrt{-1} \partial_{B}^{*} .
$$

This implies $\Delta_{B}=2 \Delta_{B}^{\prime}=2 \Delta_{B}^{\prime \prime}$, and hence, we have the Hodge structure

$$
\mathcal{H}_{B}^{r}(M) \otimes \mathbb{C}=\bigoplus_{p+q=r} \mathcal{H}_{B}^{p, q}(M) \quad \text { and } \quad \overline{\mathcal{H}_{B}^{p, q}(M)}=\mathcal{H}_{B}^{q, p}(M)
$$

where $\mathcal{H}_{B}^{p, q}(M)=\operatorname{ker} \Delta_{B_{\mid A_{B}^{p, q}(M)}^{\prime}}^{\prime}=\operatorname{ker} \Delta_{B_{\mid A}^{p, q}(M)}^{\prime \prime}$

\subsection{Twisted basic cohomology}

Let $\phi \in A_{B}^{1}(M) \otimes \mathbb{C}$ be a closed basic 1-form. Then $\left(A_{B}^{*}(M) \otimes \mathbb{C}, d_{\phi}\right)$ is a cochain complex. Denote by $H_{B}^{*}(M, \phi)$ the cohomology of this complex. It is known that there exists a subtorus $\mathcal{T} \subset \operatorname{Isom}(M, g)$ such that $A_{B}^{*}(M) \otimes \mathbb{C} \subset\left(A^{*}(M) \otimes \mathbb{C}\right)^{\mathcal{T}}$, and we have the exact sequence of complexes

$$
0 \longrightarrow A_{B}^{*}(M) \otimes \mathbb{C} \longrightarrow\left(A^{*}(M) \otimes \mathbb{C}\right)^{\mathcal{T}} \longrightarrow A_{B}^{*-1}(M) \otimes \mathbb{C} \longrightarrow 0
$$

for the usual differential $d$ (see [4, Section 7.2.1]). We can say that this is also exact for twisted differential $d_{\phi}$. Hence, taking the long exact sequence, we have the exact sequence

$$
0 \longrightarrow H_{B}^{1}(M, \phi) \longrightarrow H^{1}(M, \phi) \longrightarrow H_{B}^{0}(M, \phi)
$$


We can easily check

$$
H_{B}^{0}(M, \phi)=H^{0}(M, \phi)=0
$$

and hence we have:

\section{Lemma 3.2}

$$
H_{B}^{1}(M, \phi) \cong H^{1}(M, \phi) .
$$

Consider the formal adjoint $(\phi \wedge)_{B}^{*}: A_{B}^{r}(M) \otimes \mathbb{C} \rightarrow A_{B}^{r-1}(M) \otimes \mathbb{C}$ of the operator $\phi \wedge$ for the restricted Hermitian inner product on $A_{B}^{*}(M) \otimes \mathbb{C}$. Then we have

$$
(\phi \wedge)_{B}^{*}=*_{T}(\bar{\phi} \wedge) *_{T}
$$

(see the proof of [14, Corollary 2.3]). Taking the formal adjoint $(\phi \wedge)^{*}: A^{r}(M) \otimes \mathbb{C} \rightarrow$ $A^{r-1}(M) \otimes \mathbb{C}$ on the usual de Rham complex $A^{*}(M) \otimes \mathbb{C}$, we have

$$
(\phi \wedge)_{\mid A^{*}(M) \otimes \mathbb{C}}^{*}=(\phi \wedge)_{B}^{*} .
$$

Let $\delta_{B, \phi}=\delta_{B}+(\phi \wedge)^{*}, \Delta_{B, \phi}=d_{\phi} \delta_{B, \phi}+\delta_{B, \phi} d_{\phi}$ and $\operatorname{ker} \Delta_{B, \phi_{\mid A}(M) \otimes \mathbb{C}}=\mathcal{H}_{B}^{r}(M, \phi)$. As in $[9,11]$ and [18], we have the Hodge decomposition

$$
A_{B}^{r}(M) \otimes \mathbb{C}=\mathcal{H}_{B}^{r}(M, \phi) \oplus \operatorname{im} d_{\phi_{\mid A_{B}^{r}(M) \otimes \mathbb{C}}} \oplus \operatorname{im} \delta_{\phi_{\mid A_{B}^{r}(M) \otimes \mathbb{C}}} .
$$

Consider the double complex $\left(A_{B}^{*, *}(M), \partial_{B}, \bar{\partial}_{B}\right)$. For a $(1,0)$-basic form $\theta$, considering the operators $\theta \wedge: A_{B}^{p, q}(M) \rightarrow A_{B}^{p+1, q}(M)$ and $\Lambda: A_{B}^{p, q}(M) \rightarrow A_{B}^{p-1, q-1}(M)$, as the local argument for the Kähler identities; see, e.g., [19, Lemma 6.6]; we have the following identity.

\section{Lemma 3.3}

$$
\Lambda(\theta \wedge)-(\theta \wedge) \Lambda=-\sqrt{-1} *_{T}(\theta \wedge) *_{T} \quad \text { and } \quad \Lambda(\bar{\theta} \wedge)-(\bar{\theta} \wedge) \Lambda=\sqrt{-1} *_{T}(\bar{\theta} \wedge) *_{T} .
$$

Let $\phi \in \mathcal{H}_{B}^{1}(M) \otimes \mathbb{C}$. By $\mathcal{H}_{B}^{1}(M)=\mathcal{H}_{B}^{1,0}(M) \oplus \mathcal{H}_{B}^{0,1}(M)$, we can take unique $\theta_{1}$, $\theta_{2} \in \mathcal{H}_{B}^{1,0}(M)$ such that $\phi=\theta_{1}+\bar{\theta}_{1}+\theta_{2}-\bar{\theta}_{2}$. Define

$$
\partial_{B, \theta_{1}, \theta_{2}}=\partial_{B}+\theta_{2} \wedge+\bar{\theta}_{1} \wedge \quad \text { and } \quad \bar{\partial}_{B, \theta_{1}, \theta_{2}}=\bar{\partial}_{B}-\bar{\theta}_{2} \wedge+\theta_{1} \wedge .
$$

By $\mathcal{H}_{B}^{p, q}(M)=\operatorname{ker} \Delta_{B_{\mid A_{B}^{p, q}(M)}^{\prime}}^{\prime}=\operatorname{ker} \Delta_{B_{\mid A_{B}^{p, q}(M)}^{\prime \prime}},\left(A_{B}^{*}(M) \otimes \mathbb{C}, \partial_{B, \theta_{1}, \theta_{2}}\right)$ and $\left(A_{B}^{*}(M) \otimes\right.$ $\left.\mathbb{C}, \bar{\partial}_{B, \theta_{1}, \theta_{2}}\right)$ are cochain complexes. Denote by $H_{B}^{*}\left(M, \theta_{1}, \theta_{2}\right)$ the cohomology of $\left(A_{B}^{*}(M) \otimes\right.$ $\left.\mathbb{C}, \bar{\partial}_{B, \theta_{1}, \theta_{2}}\right)$. As similar to [13, Lemma 2.1], we obtain the following lemma.

Lemma 3.4 For any $t \in \mathbb{R}^{*}$, we have

$$
\operatorname{dim} H_{B}^{*}\left(M, \theta_{1}, \theta_{2}\right)=\operatorname{dim} H_{B}^{*}\left(M, t \theta_{1}, \theta_{2}\right) .
$$

Consider the formal adjoint

$$
\partial_{B, \theta_{1}, \theta_{2}}^{*}=\partial_{B}^{*}+\left(\theta_{2} \wedge\right)^{*}+\left(\bar{\theta}_{1} \wedge\right)^{*} \quad \text { and } \quad \bar{\partial}_{B, \theta_{1}, \theta_{2}}^{*}=\bar{\partial}_{B}^{*}-\left(\bar{\theta}_{2} \wedge\right)^{*}+\left(\theta_{1} \wedge\right)^{*} .
$$

Since we have $\left(\theta_{i} \wedge\right)^{*}=*_{T}\left(\bar{\theta}_{i} \wedge\right) *_{T}$, by the Lemma 3.1 and 3.3, we obtain the following Kähler identity (cf. [1, Section 1.2], [17, page 15]).

\section{Lemma 3.5}

$$
\Lambda \partial_{B, \theta_{1}, \theta_{2}}-\partial_{B, \theta_{1}, \theta_{2}} \Lambda=\sqrt{-1} \bar{\partial}_{B, \theta_{1}, \theta_{2}}^{*} \quad \text { and } \quad \Lambda \bar{\partial}_{B, \theta_{1}, \theta_{2}}-\bar{\partial}_{B, \theta_{1}, \theta_{2}} \Lambda=-\sqrt{-1} \partial_{B, \theta_{1}, \theta_{2}}^{*} \text {. }
$$


Define

$$
\begin{gathered}
\Delta_{B, \theta_{1}, \theta_{2}}^{\prime}=\partial_{B, \theta_{1}, \theta_{2}} \partial_{B, \theta_{1}, \theta_{2}}^{*}+\partial_{B, \theta_{1}, \theta_{2}}^{*} \partial_{B, \theta_{1}, \theta_{2}} \text { and } \\
\Delta_{B, \theta_{1}, \theta_{2}}^{\prime \prime}=\bar{\partial}_{B, \theta_{1}, \theta_{2}} \bar{\partial}_{B, \theta_{1}, \theta_{2}}^{*}+\bar{\partial}_{B, \theta_{1}, \theta_{2}}^{*} \bar{\partial}_{B, \theta_{1}, \theta_{2} .} .
\end{gathered}
$$

Then Lemma 3.5 implies $\Delta_{B, \phi}=2 \Delta_{B, \theta_{1}, \theta_{2}}^{\prime \prime}=2 \Delta_{B, \theta_{1}, \theta_{2}}^{\prime}$. Denote $\mathcal{H}_{B}^{r}\left(M, \theta_{1}, \theta_{2}\right)=$

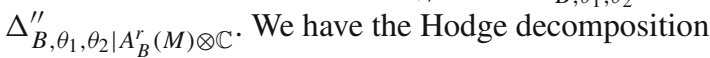

$$
A_{B}^{r}(M) \otimes \mathbb{C}=\mathcal{H}_{B}^{r}\left(M, \theta_{1}, \theta_{2}\right) \oplus \operatorname{im} \bar{\partial}_{B, \theta_{1}, \theta_{2} \mid A_{B}^{r-1}(M) \otimes \mathbb{C}} \oplus \operatorname{im} \bar{\partial}_{B, \theta_{1}, \theta_{2} \mid A_{B}^{r+1}(M) \otimes \mathbb{C}}^{*}
$$

Hence, we obtain an isomorphism

$$
H_{B}^{r}(M, \phi) \cong H_{B}^{r}\left(M, \theta_{1}, \theta_{2}\right) .
$$

By Lemma 3.2 and 3.4, we have the following result.

Theorem 3.6 For any $t \in \mathbb{R}^{*}$, we have the equation

$$
\operatorname{dim} H^{1}(M, \phi)=\operatorname{dim} H^{1}\left(M, t \theta_{1}+t \bar{\theta}_{1}+\theta_{2}-\bar{\theta}_{2}\right) .
$$
1.1 .

Since we have isomorphisms $H^{1}(M, \mathbb{C}) \cong H_{B}^{1}(M, \mathbb{C}) \cong \mathcal{H}_{B}^{1}(M) \otimes \mathbb{C}$, we obtain Theorem

\section{Proof of Corollary 1.3}

Let $G$ be a simply connected solvable Lie group and $N$ be the nilradical (i.e., maximal connected nilpotent normal subgroup) of $G$. Denote by $\mathfrak{g}$ the Lie algebra of $G$. Then we can take a simply connected nilpotent subgroup $C \subset G$ such that $G=C \cdot N$ (see [8, Proposition 3.3]). Since $C$ is nilpotent, the map

$$
\Phi: C \ni c \mapsto\left(\operatorname{Ad}_{c}\right)_{s} \in \operatorname{Aut}(\mathfrak{g})
$$

is a diagonalizable representation where $\left(\operatorname{Ad}_{c}\right)_{s}$ is the semi-simple part of the adjoint operator $\operatorname{Ad}_{c}$ (see [12]). We take a diagonalization $\Phi=\operatorname{diag}\left(\alpha_{1}, \ldots, \alpha_{n}\right)$ where $\alpha_{1}, \ldots, \alpha_{n}$ are $\mathbb{C}$ valued characters of $C$. Since the adjoint representation Ad on the nilradical $N$ is unipotent and we have an isomorphism $G / N \cong C / C \cap N, \alpha_{1}, \ldots, \alpha_{n}$ are considered as characters of $G$. For each $g \in G, \alpha_{i}(g)$ is an eigenvalue of $\operatorname{Ad}_{g}$.

Suppose $G$ admits a lattice $\Gamma$. We consider the solvmanifold $G / \Gamma . G / \Gamma$ is an aspherical manifold with the fundamental group $\Gamma$. In [13], the set $\mathcal{J}_{1}(\Gamma)$ was studied. The author proved that $\mathcal{J}_{1}(\Gamma)$ is a finite set ([13, Corollary 5.9]) and if one of the characters $\alpha_{1}, \ldots, \alpha_{n}$ is nonunitary, then there exists a non-unitary character $\rho \in \mathcal{C}(\Gamma)$ of $\Gamma$ such that $\rho \in \mathcal{J}_{1}(\Gamma)$ ([13, Corollary 5.10]). We suppose that $\Gamma$ can be the fundamental group of a compact Sasakian manifold. Then by Corollary 1.2, characters $\alpha_{1}, \ldots, \alpha_{n}$ are all unitary. Hence, for any $g \in G$, all eigenvalues of $\operatorname{Ad}_{g}$ are unitary. In this case, a lattice $\Gamma$ of $G$ is virtually nilpotent (see [3, Chapter IV.5]). It is known that every polycyclic group contains a lattice of some simply connected solvable Lie group as a finite index normal subgroup (see [15, Theorem 4.28]). Hence Corollary 1.3 follows.

Acknowledgments The author would like to thank Xiaoyang Chen for introducing to his results. This research is supported by JSPS Research Fellowships for Young Scientists. 


\section{References}

1. Angella, D., Kasuya, H.: Hodge theory for twisted differentials. Complex Manifolds 1, 64-85 (2014)

2. Arapura, D., Nori, M.: Solvable fundamental groups of algebraic varieties and Kähler manifolds. Compositio Math. 116(2), 173-188 (1999)

3. Auslander, L.: An exposition of the structure of solvmanifolds, I. Algebraic theory. Bull. Am. Math. Soc. 79(2), 227-261 (1973)

4. Boyer, C.P., Galicki, K.: Sasakian geometry. Oxford mathematical monographs. Oxford University Press, Oxford (2008)

5. Campana, F.: Quotients résoluble ou nilpotents des groupes de Kähler orbifoldes. Manuscr. Math. 135(12), 117-150 (2011)

6. Cappelletti-Montano, B., De Nicola, A., Marrero, J.C., Yudin, I.: Sasakian nilmanifolds. Int. Math. Res. Not. (2014). doi:10.1093/imrn/rnu144

7. Chen, X.: On the fundamental groups of compact Sasakian manifolds. Math. Res. Lett. 20(1), 27-39 (2013)

8. Dekimpe, K.: Semi-simple splittings for solvable Lie groups and polynomial structures. Forum Math. 12(1), 77-96 (2000)

9. El Kacimi-Alaoui, A.: Opérateurs transversalement elliptiques sur un feuilletage riemannien et applications. Compos. Math. 73(1), 57-106 (1990)

10. Hasegawa, K.: A note on compact solvmanifolds with Kähler structures. Osaka J. Math. 43(1), 131-135 (2006)

11. Kamber, F.W., Tondeur, P.: de Rham-Hodge theory for Riemannian foliations. Math. Ann. 277(3), 415431 (1987)

12. Kasuya, H.: de Rham and Dolbeault cohomology of solvmanifolds with local systems. Math. Res. Lett. 21(4), 781-805 (2014)

13. Kasuya, H.: Flat bundles and Hyper-Hodge decomposition on solvmanifolds. Int. Math. Res. Not. IMRN. arXiv: $1309.4264 \mathrm{v} 1$ [math.DG]

14. Park, E., Richardson, K.: The basic Laplacian of a Riemannian foliation. Am. J. Math. 118(6), 1249-1275 (1996)

15. Raghnathan, M.S.: Discrete subgroups of Lie Groups. Springer, New York (1972)

16. Saitô, M.: Sur certains groupes de Lie résolubles II. Sci. Papers Coll. Gen. Educ. Univ. Tokyo 7, 157-168 (1957)

17. Simpson, C.T.: Higgs bundles and local systems. Inst. Hautes Études Sci. Publ. Math. 75, 5-95 (1992)

18. Tievsky, A.M.: Analogues of Kähler Geometry on Sasakian Manifolds. Ph.D. Thesis, Massachusetts Institute of Technology (2008). http://dspace.mit.edu/handle/1721.1/45349

19. Voisin, C.: Hodge Theory and Complex Algebraic Geometry, I. Cambridge Studies in Advanced Mathematics, 76. Cambridge University Press, Cambridge (2002) 